\title{
Afatinib for the treatment of advanced non-small-cell lung cancer harboring an epidermal growth factor receptor exon 18 E709_T710delinsD mutation: a case report
}

\author{
Lander Van Acker ${ }^{*}$, Dieter Stevens, Karim Vermaelen and Veerle Surmont
}

\begin{abstract}
Background: The discovery of epidermal growth factor receptor oncogenic driver mutations has changed the therapeutic landscape of advanced non-small cell lung cancer in the past decade. Since the introduction of nextgeneration sequencing, uncommon epidermal growth factor receptor mutations are more frequently discovered. Because seldom evaluated in clinical trials, their clinical significance and response on tyrosine kinase inhibitors are less well known.

Case presentation: A 58-year-old Caucasian woman with no smoking history presented with advanced non-small cell lung cancer. Liver biopsy revealed an adenocarcinoma with a programmed death ligand-1 tumor proportion score of 30\% and no common oncogenic driver mutations. A combination of chemotherapy and immunotherapy was started as first-line treatment. However, treatment was ceased after 18 weeks because of immune-related renal failure and disease progression. In the meantime, the next-generation sequencing results of the liver biopsy had revealed an exon 18 E709_T710delinsD mutation. Therefore, afatinib was administered, which was moderately tolerated with grade 2 paronychia and acneiform skin eruption. After 6 months, a partial response with ongoing decrease of the liver metastasis was retained.

Conclusion: Because of the lack of clinical trials, tumor heterogeneity, and a tyrosine kinase inhibitor affinity related to the different mutation types, it is difficult to predict the clinical outcome of tyrosine kinase inhibitor in uncommon mutations. Therefore, a therapeutic trial with tyrosine kinase inhibitor has to be considered, but the expected clinical response is lower than for common mutations.
\end{abstract}

Keywords: $\mathrm{NSCLC}_{1}$, EGFR exon 18, E709_T710delinsD mutation ${ }_{3}$, Afatinib ${ }_{4}$, Case report

\section{Introduction}

The discovery of epidermal growth factor receptor (EGFR) oncogenic driver mutations preluded a new therapeutic area for lung cancer treatment in the past decade. EGFR mutations are present in $30-60 \%$ of Asian and $10-20 \%$ of Caucasian patients with non-small-cell lung

*Correspondence: lander.vanacker@uzgent.be

University Hospital Ghent, Ghent, Belgium cancer (NSCLC). These mutations are more frequently observed in female patients, patients with lung adenocarcinoma, and never smokers. Three generations of tyrosine kinase inhibitors (TKI) have already been developed for common EGFR mutations and showed clinical efficacy in several phase III trials as first- and second-line treatment for metastatic non-small-cell lung adenocarcinoma [1, 2]. Recent data also revealed favorable disease-free survival with TKIs as an adjuvant treatment in resectable disease [3]. original author(s) and the source, provide a link to the Creative Commons licence, and indicate if changes were made. The images or other third party material in this article are included in the article's Creative Commons licence, unless indicated otherwise in a credit line to the material. If material is not included in the article's Creative Commons licence and your intended use is not permitted by statutory regulation or exceeds the permitted use, you will need to obtain permission directly from the copyright holder. To view a copy of this licence, visit http://creativecommons.org/licenses/by/4.0/. The Creative Commons Public Domain Dedication waiver (http://creativeco mmons.org/publicdomain/zero/1.0/) applies to the data made available in this article, unless otherwise stated in a credit line to the data. 
Afatinib, a second-generation TKI, has showed to improve the progression-free survival (PFS) in patients with common mutations such as exon 19 deletions and exon 21 Lco858R point mutations, accounting for approximately $85 \%$ of the EGFR mutations [4-6]. Its importance has decreased since the introduction of osimertinib with better results on PFS and toxicity in firstand second-line treatment $[2,7]$.

The clinical significance of uncommon EGFR mutations is less known. Few clinical data, mostly based on case reports, reported a favorable response on afatinib in EGFR exon 18 mutations [8]. We present a case report of a patient with advanced stage lung adenocarcinoma with an E709_T710delinsD mutation.

\section{Case presentation}

A 58-year-old Caucasian woman presented with a chronic cough and progressive dyspnea since 3 months. Her medical history included urolithiasis and a multinodular goiter, treated with a left hemithyroidectomy and thyroid hormone substitution. She had no antecedents of pulmonary disease, and there was no active or passive tobacco exposition. Her sister was treated for breast cancer, but no other family history of cancer was mentioned. Her World Health Organization (WHO) performance score was 1 . Clinical examination revealed decreased breath sounds at the base of the right lung. Computed tomography $(\mathrm{CT})$ revealed a necrotic mass in the apical segment of the right lower lobe, extending to the right hilum and encasing the right main and lower lobe bronchus. There were several enlarged mediastinal lymph nodes and right-sided pleural fluid. Positron emission tomography (PET) visualized a fluorodeoxyglucose (FDG)-avid liver lesion in segment 4b (Fig. 1). Pathologic diagnosis of an adenocarcinoma [thyroid transcription factor 1 (TTF1) positive] was confirmed by endobronchial ultrasound and CT-guided liver biopsy. The malign process was staged as a cT2aN3M1b or stage IVb adenocarcinoma (TNM 8th edition). The programmed death ligand-1 (PD-L1) tumor proportion score was 30\% using the Roche SP 263 antibody assay. Anaplastic lymphoma kinase (ALK)- and reactive oxygen species 1 (ROS1)- immunohistochemistry was negative. Molecular analysis, performed by next-generation sequencing (NGS, SeqCap) revealed an EGFR mutation in exon 18, E709_T710delinsD. Since the result of the NGS was not yet available, the patient was included in a phase III randomized controlled trial with carboplatin, pemetrexed, and experimental immunotherapy with a combined transforming growth factor beta (TGF- $\beta$ ) and PD-L1 inhibitor. After two cycles, a partial response (PR) was observed with a stable situation after four cycles. Maintenance study immunotherapy was continued, but after two cycles there was disease progression of the primary tumor and the liver metastasis. Furthermore, the patient was hospitalized with grade 3 acute kidney injury, caused by an immune-mediated interstitial nephritis. The treatment was ceased, and renal function was restored after corticosteroid administration during 3 months. Two months later, afatinib $40 \mathrm{mg}$ was initiated because of progressive disease (PD). This treatment was moderately tolerated with grade 2 paronychia and a grade 2 acneiform skin eruption, treated with tetracycline and topical therapy. After 6 months, a PR with ongoing decrease of the liver metastasis was retained (Fig. 2). Because of persistent grade 2 skin toxicity, the dose of afatinib was reduced to $30 \mathrm{mg}$.

\section{Discussion}

The most common EGFR mutations are found in exons 19-21, with exon 19 deletions (45\%) and exon 21 L858R point mutations (40\%) being responsible for $85-90 \%$ of the EGFR-mutated NSCLC [6, 9, 10]. Inhibition of this mutated EGFR with the third-generation TKI osimertinib as first-line treatment resulted in an objective response rate (ORR) of about $80 \%$ of the patients, with
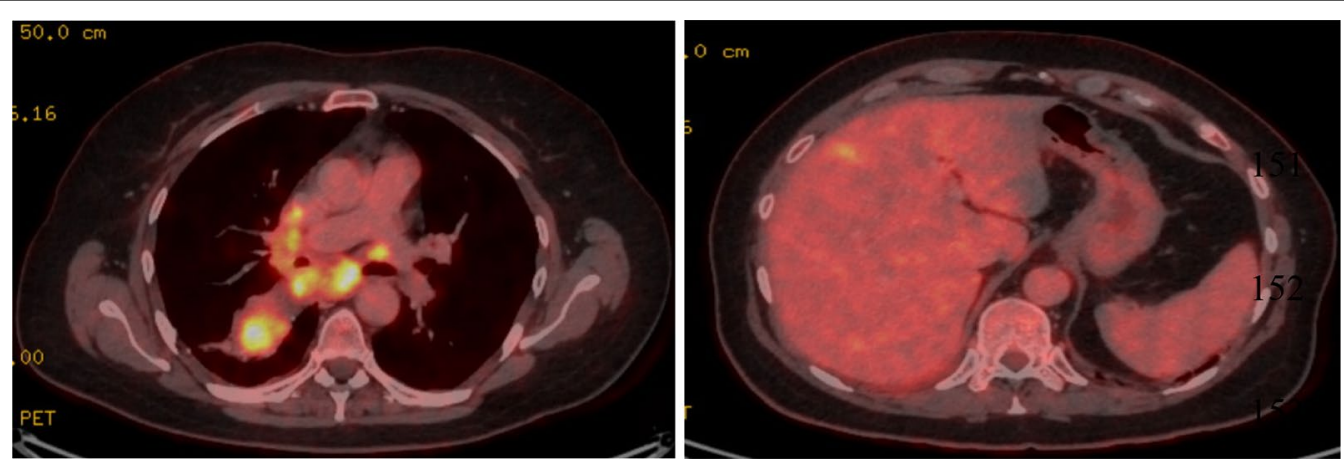

Fig. 1 Positron Emission Tomography-Computed Tomography (PET-CT) at presentation 


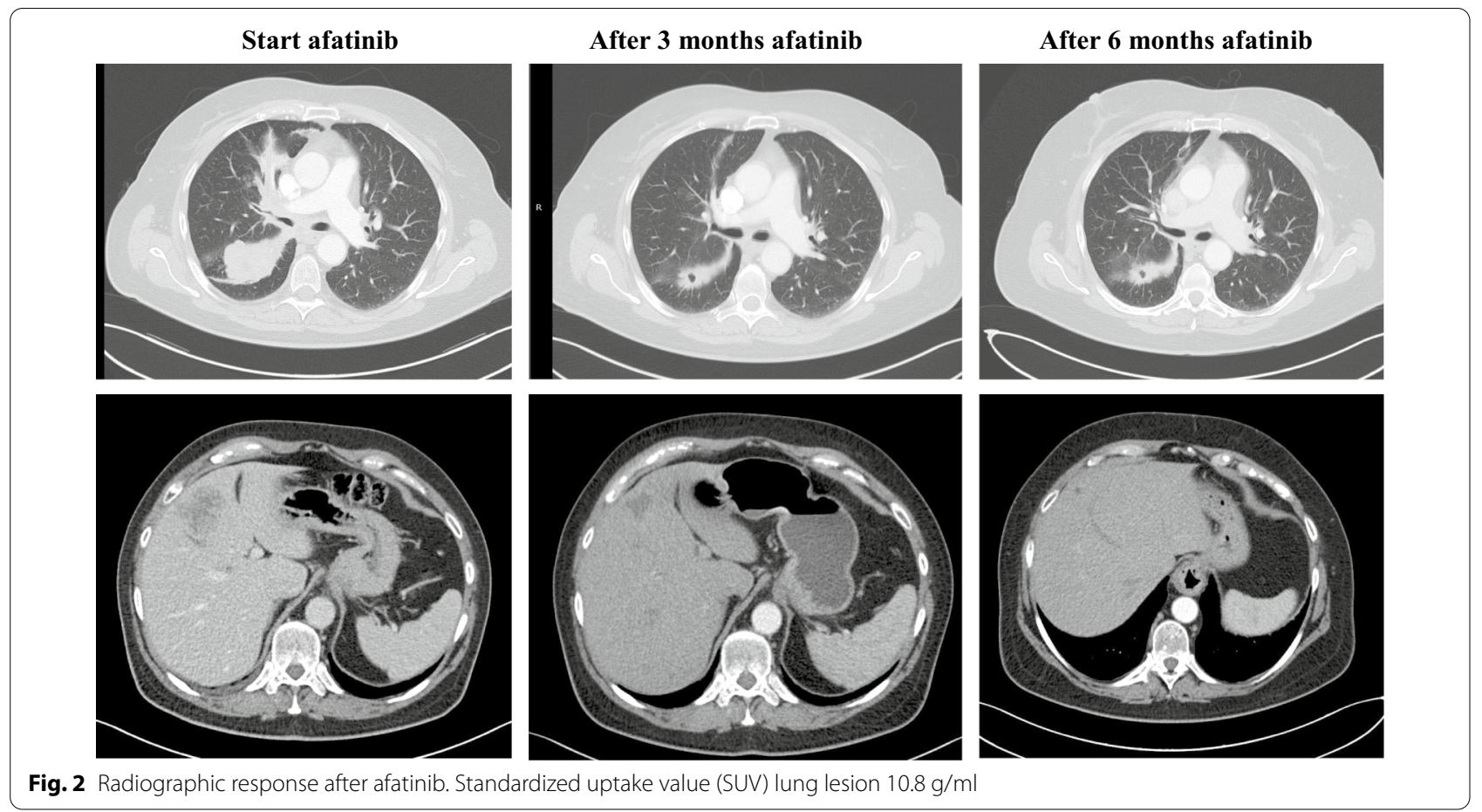

a PFS of 18.9 months and an overall survival (OS) of 38.6 months in the FLAURA trial. T790M (exon 20) is a rather uncommon mutation, but its clinical significance is well known as the cause of TKI acquired resistance in almost $50-60 \%$ of the patients after first- or second-line TKI treatment $[2,11]$.

The other, so-called uncommon, mutations are present in $7-23 \%$ of the EGFR-mutated NSCLC. They are more heterogeneous (about 600 known variations), and the detection of new variants is still ongoing. Exon 20 insertions (6\%), exon 18 G719X (3\%), exon 21 L861Q (1\%), S768I (1\%), and exon 19 insertions (0.6\%) are more frequently reported mutations, noted as major uncommon mutations [8]. They are less gender dependent and also occur in (ex-)smokers $[11,12]$. However, the prevalence of uncommon mutations will probably change in the next coming years, due to the broader application of NGS for molecular tumor profiling. Until recently, EGFR mutation testing was mostly based on commercial genotyping assays (COBAS, Therascreen) with heterogenic targets. For example, most kits only included exon 18 G719 point mutations and no E709_T70delinsD mutations [8].

Exon 18 mutations account for $4.1 \%$ of all EGFR mutations following the catalogue of somatic mutations in cancer (COSMIC) database $[11,13]$. In a large French study, a frequency of 5.5\% was found [14]. Two types are described: a deletion-insertion or a point mutation. G719X (mostly G719A), followed by E709K, were the more frequently observed point mutations.
E709-T710delinsD was the most common deletion, but only accounting for less than $0.1 \%$ of the EGFR mutations $[9,15]$. Seventeen cases of an E709_T70delinsD mutation have been reported in the COSMIC database and 27 in MyCancergenome.org [13, 16].

Compound mutations, consisting of a common and an uncommon mutation, are more frequent than single mutations, harboring $2-25 \%$ of the EGFR mutationpositive lung cancers. The prognosis of patients with these mutations is reduced in comparison with two common mutations, but remains higher than for uncommon mutations [6].

Less is known about the clinical relevance and therapeutic response when targeting single uncommon mutations because they were excluded in most trials. Moreover, there are no specific clinical trials exploring the effect of TKIs on EGFR exon 18 mutations. Small numbers of patients with uncommon mutations $(+/-10 \%)$ were included in trials with gefitinib (Iressa Pan-Asia Study) and afatinib (LUX-Lung 3 and 6), but the predictive value could not be determined owing to the small study populations $[8,11]$. In a post hoc analysis of afatinib, performed by Yang et al., there was demonstrated clinical activity against the major uncommon mutations (exon 18 G719X, exon 21 L861Q, and S768I). The response rate for the sensitizing exon 18 G719X mutations was lower than for common mutations, but exceeded $50 \%(63.4 \%)$ with a time to treatment failure of 14.7 months (8.1-17.1 months) in treatment-naïve 
patients [8]. Recently, some evidence for osimertinib in uncommon mutations was published by Cho et al. For example, an objective response of $53 \%$ with a PFS of 8.2 months was found for exon 18 G179X mutations [17].

In a French retrospective study, the PFS for exon 18 mutations after TKI was 14.6 months against 5.8 months after chemotherapy. The OS was 12.2 months, influenced by the fact that one-third of the people did not manage to receive a TKI after chemotherapy. In conclusion, the authors proposed a trial with TKI as first-line therapy. However, the therapeutic response was very heterogeneous among the mutation variants and higher for proximal localized mutations (G719X and E709X point mutations), so the effect remained difficult to predict [14].

There are even fewer data available for the specific exon 18 E709_T70delinsD mutation [8]. An in vitro study, performed by Kobayashi et al. explored the role of exon 18 mutations, inclusive E709_T70delinsD, and appointed these as oncogenic driver mutations. They observed a higher sensitivity for second-generation TKIs in comparison with first- and third-generation TKIs [9]. The underlying mechanism seemed to be a variable affinity to the targeted kinase, with the highest affinity for afatinib $[8$, 9].

Because of scarce evidence, treatment responses of afatinib in patients with an E709_T70delinsD mutation are mostly based on several case series. On the website https://www.uncommonegfrmutations.com clinical cases are collected to get a better insight in the clinical outcome. An overview of the published case reports in literature is presented in Table 1 with a total of 14 cases. All patients had a history of an adenocarcinoma without a gender predominance. They were more frequent of Asian origin, and most of them had no tobacco history. Seven patients (50\%), including all cases with afatinib, had a PR. One case showed a good intracranial response, although combined with bevacizumab. Data of OS are lacking and are difficult to compare because patients had undergone multiple previous treatment lines $[4,9,10,14,15,18-22]$.

\section{Conclusion}

This case report is an example of a favorable response in an EGFR-mutated NSCLC with a "minor" uncommon mutation successfully treated with afatinib. Because of the limited evidence and the absence of clinical trials, no robust treatment recommendations can be made. EGFRTKI therapy, with most reports available for afatinib, can be an option in patients with exon 18 mutations, but the expected clinical response is lower than for common mutations. However, tumor heterogeneity and a TKI affinity related to the different mutation types complicate the prediction of the clinical outcome of TKI in uncommon mutations. In conclusion, a therapeutic trial with TKI has to be considered.

\footnotetext{
Abbreviations

EGFR: Epidermal growth factor receptor; NSCLC: Non-small-cell lung cancer; NGS: Next-generation sequencing; TKI: Tyrosine kinase inhibitor; PD-L1: Programmed death ligand 1; PFS: Progression-free survival; WHO: World Health Organization; CT: Computed tomography; PET: Positron emission tomography FDG: Fluorodeoxyglucose; TTF1: Thyroid transcription factor 1; ALK: Anaplastic lymphoma kinase; ROS1: Reactive oxygen species 1; PR: Partial response; PD: Progressive disease; ORR: Objective response rate; OS: Overall survival; COSMIC: Catalogue of somatic mutations in cancer.
}

Table 1 Summary of the case reports with an epidermal growth factor receptor exon 18 E709_T710delinsD [4, 9, 10, 14, 15, 18-22]

\begin{tabular}{|c|c|c|c|c|c|c|c|c|c|}
\hline Case report & Ethnicity & Age & Gender & Smoking & Histology & TKI & Response & PFS (m) & OS (m) \\
\hline Wu et al. & Asian & 61 & $\mathrm{~F}$ & No & $A D$ & Gefitinib & SD & 5.1 & 22.7 \\
\hline Wuetal. & Asian & 65 & $M$ & Yes & $A D$ & Gefitinib & PD & 0.9 & 11.1 \\
\hline Kobayashi et al. & Asian & 63 & $M$ & $\mathrm{~N} / \mathrm{A}$ & $A D$ & $\begin{array}{l}\text { Erlotinib** } \\
\text { Afatinib }\end{array}$ & $\begin{array}{l}\text { SD } \\
\text { PR }\end{array}$ & $\begin{array}{l}N / A \\
N / A\end{array}$ & N/AN/A \\
\hline Ackerman et al. & Non-Asian & 88 & $F$ & No & $A D$ & Erlotinib & $P R$ & 6 (stop) & $\mathrm{N} / \mathrm{A}$ \\
\hline Leduc et al. & Non-Asian & $\mathrm{N} / \mathrm{A}$ & $\mathrm{N} / \mathrm{A}$ & $\mathrm{N} / \mathrm{A}$ & $\mathrm{N} / \mathrm{A}$ & $\mathrm{N} / \mathrm{A}$ & $\mathrm{N} / \mathrm{A}$ & 3 & $\mathrm{~N} / \mathrm{A}$ \\
\hline Ibrahim et al. & Non-Asian & 52 & $F$ & No & $A D$ & Afatinib & $P R$ & $\mathrm{~N} / \mathrm{A}$ & $\mathrm{N} / \mathrm{A}$ \\
\hline Iwamoto et al. & Asian & 56 & $F$ & No & $A D$ & Afatinib & PR & 7 & $\mathrm{~N} / \mathrm{A}$ \\
\hline Anetal. & Asian & 71 & $M$ & Yes & $A D$ & Afatinib ${ }^{* * *}$ & PR & 11 & $>21$ \\
\hline Klughammer et al. & Asian & 50 & $\mathrm{~F}$ & No & $A D$ & Erlotinib* & PD & 38 & 52 \\
\hline Mehta et al. & Asian & 61 & M & $\mathrm{N} / \mathrm{A}$ & $A D$ & Erlotinib* & $P R$ & 7 & $\mathrm{~N} / \mathrm{A}$ \\
\hline Sousa et al. & Non-Asian & $\mathrm{N} / \mathrm{A}$ & N/A & N/A & $\mathrm{N} / \mathrm{A}$ & Gefitinib & PD & 3 & 24 \\
\hline Sousa et al. & Non-Asian & $\mathrm{N} / \mathrm{A}$ & $\mathrm{N} / \mathrm{A}$ & N/A & N/A & Erlotinib* & PD & 4 & 26 \\
\hline Sousa et al. & Non-Asian & $\mathrm{N} / \mathrm{A}$ & $\mathrm{N} / \mathrm{A}$ & $\mathrm{N} / \mathrm{A}$ & $\mathrm{N} / \mathrm{A}$ & Erlotinib* & PD & 3 & 18 \\
\hline This case & Non-Asian & 58 & F & No & $A D$ & Afatinib* & PR & $>6$ & N/A \\
\hline
\end{tabular}

$A D$ adenocarcinoma, $P R$ partial response, $S D$ stable disease, $P D$ progressive disease, $N / A$ not available, ${ }^{*}$ second-line treatment; ${ }^{* *}$ intolerance; ${ }^{* * *}+$ bevacizumab 


\section{Acknowledgements \\ Not applicable.}

\section{Authors' contributions}

LVA was the primary author of the manuscript. DS and VS provided comments and reviewed the manuscript. KV was involved in the clinical follow-up of the patient. All authors read and approved the final manuscript.

\section{Funding}

Not applicable.

\section{Availability of data and materials}

Not applicable.

\section{Declarations}

\section{Ethics approval and consent to participate}

This publication was approved by the local ethics committee (University Hospitals Ghent)

\section{Consent for publication}

Written informed consent was obtained from the patient for publication of this case report and any accompanying images. A copy of the written consent is available for review by the Editor-in-Chief of this journal.

\section{Competing interests}

The authors declare that the research was conducted in the absence of any commercial or financial relationships that could be construed as a potential conflict of interest.

Received: 19 February 2021 Accepted: 1 July 2021

Published online: 22 November 2021

\section{References}

1. Greenhalgh J, Dwan K, Boland A, et al. First-line treatment of advanced epidermal growth factor receptor (EGFR) mutation positive nonsquamous non-small cell lung cancer. Cochrane Database Syst Rev. 2016:5:CD1010383.

2. Ramalingam SS, Vansteenkiste J, Planchard D, FLAURA Investigators, et al. Overall survival with osimertinib in untreated, EGFR-mutated advanced NSCLC. N Engl J Med. 2020;382(1):41-50

3. Wu YL, Tsuboi M, He J, et al. Osimertinib in resected EGFR-mutated nonsmall-cell lung cancer. N Engl J Med. 2020;383(18):1711-23.

4. Wu J-Y, Yu C-J, Chang Y-C, et al. Effectiveness of tyrosine kinase inhibitors on "uncommon" epidermal growth factor receptor mutations of unknown clinical significance in non-small cell lung cancer. Clin Cancer Res. 2011;17(11):3812-21.

5. Sequist LV, Yang JC, Yamamoto N, et al. Phase III study of afatinib or cisplatin plus pemetrexed in patients with metastatic lung adenocarcinoma with EGFR mutations. J Clin Oncol. 2013;31(27):3327-34.

6. Kutsuzawa N, Takahashi F, Tomomatsu K, et al. Successful treatment of a patient with lung adenocarcinoma harboring compound EGFR gene mutations, G719X and S768I, with Afatinib. Tokai J Exp Clin Med. 2020;45(3):113-6.
7. MokTS, Wu Y-L, Ahn M-J, AURA3 Investigators, et al. Osimertinib or platinum-pemetrexed in EGFR T790M-positive lung cancer. N Engl J Med. 2017:376(7):629-40.

8. Yang JC, Schuler M, Popat S, et al. Afatinib for the treatment of NSCLC harboring uncommon EGFR mutations: a database of 693 cases. J Thorac Oncol. 2020;15(5):803-15.

9. Kobayashi Y, Togashi Y, Yatabe Y, et al. EGFR exon 18 mutations in lung cancer: molecular predictors of augmented sensitivity to afatinib or neratinib as compared with first- or third-generation TKIs. Clin Cancer Res. 2015;21(23):5305-13.

10. Ackerman A, Goldstein MA, Kobayashi S, Costa DB. EGFR delE709_ T710insD: a rare but potentially EGFR inhibitor responsive mutation in non-small-cell lung cancer. J Thorac Oncol. 2012;7(10):e19-20.

11. O'Kane GM, Bradbury PA, Ronald Feld R, et al. Uncommon EGFR mutations in advanced non-small cell lung cancer. Lung Cancer. 2017:109:137-44

12. Pilotto S, Rossi A, Vavalà T, et al. Outcomes of first-generation EGFR-TKls against non-small-cell lung cancer harboring uncommon EGFR mutations: a post hoc analysis of the BE-POSITIVE study. Clin Lung Cancer. 2018;19(1):93-104.

13. Catalogue of somatic mutations in cancer (COSMIC). https://cancer. sanger.ac.uk. Accessed 4 February 2021.

14. Leduc C, Merlio JP, Besse B, et al. Clinical and molecular characteristics of non-small-cell lung cancer (NSCLC) harboring EGFR mutation: results of the nationwide French Cooperative Thoracic Intergroup (IFCT) program. Ann Oncol. 2017;28(11):2715-24.

15. Ibrahim U, Saqib A, Atallah JP. EGFR exon 18 delE709_T710insD mutated stage IV lung adenocarcinoma with response to afatinib. Lung Cancer. 2017:108:45-7.

16. My Cancer genome. http://mycancergenome.org. Accessed 4 February 2021.

17. Cho JH, Lim SH, An HJ, et al. Osimertinib for patients with non-small-cell lung cancer harboring uncommon EGFR mutations: a multicenter, openlabel, phase II trial (KCSG-LU15-09). J Clin Oncol. 2020;38(5):488-95.

18. Iwamoto $Y$, Ichihara $E$, Hara $N$, et al. Efficacy of afatinib treatment for lung adenocarcinoma harboring exon 18 delE709_T710insD mutation. Jpn J Clin Oncol. 2019

19. An N, Wang H, Zhu H, et al. Great efficacy of afatinib on a patient with lung adenocarcinoma harboring uncommon EGFR delE709_T710insD mutations: a case report. Onco Targets Ther. 2019;12:7399-404.

20. Klughammer B, Brugger W, Cappuzzo F, et al. Examining treatment outcomes with erlotinib in patients with advanced non-small cell lung cancer whose tumors harbor uncommon EGFR mutations. J Thorac Oncol Actions. 2016;11(4):545-55.

21. Mehta A, Batra U, Sharma M, et al. Detection of rare targetable EGFR variant in metastatic non-small cell lung carcinoma by next generation sequencing: a case report. J Clin Diag Res. 2020;14(6):GD01-3.

22. Sousa AC, Silveira C, Janeiro A, et al. Detection of rare and novel EGFR mutations in NSCLC patients: implications for treatment-decision. Lung Cancer. 2020;139:35-40

\section{Publisher's Note}

Springer Nature remains neutral with regard to jurisdictional claims in published maps and institutional affiliations. 